\title{
Molecular consequences of cystic fibrosis transmembrane regulator (CFTR) gene mutations in the exocrine pancreas
}

\author{
N Ahmed, M Corey, G Forstner, J Zielenski, L-C Tsui, L Ellis, E Tullis, P Durie
}

Gut 2003;52:1159-1164

See end of article for authors' affiliations

\section{Correspondence to:} Dr P R Durie, Division of Gastroenterology and Nutrition, The Hospital for Sick Children, 555

University Ave, Toronto,

Ontario M5G $1 \times 8$,

Canada;

peter.durie@sickkids.ca

Accepted for publication 18 March 2003

\begin{abstract}
Background and aims: We tested the hypothesis that the actual or predicted consequences of mutations in the cystic fibrosis transmembrane regulator gene correlate with the pancreatic phenotype and with measures of quantitative exocrine pancreatic function.

Methods: We assessed 742 patients with cystic fibrosis for whom genotype and clinical data were available. At diagnosis, 610 were pancreatic insufficient, 110 were pancreatic sufficient, and 22 pancreatic sufficient patients progressed to pancreatic insufficiency after diagnosis.

Results: We identified mutations on both alleles in 633 patients $(85.3 \%)$, on one allele in $95(12.8 \%)$, and on neither allele in $14(1.9 \%)$. Seventy six different mutations were identified. The most common mutation was $\Delta \mathrm{F} 508(71.3 \%)$ followed by G551D (2.9\%), G542X (2.3\%), 621+1G $\rightarrow T(1.2 \%)$, and W1282X (1.2\%). Patients were categorized into five classes according to the predicted functional consequences of each mutation. Over $95 \%$ of patients with severe class I, II, and III mutations were pancreatic insufficient or progressed to pancreatic insufficiency. In contrast, patients with mild class IV and $\mathrm{V}$ mutations were consistently pancreatic sufficient. In all but four cases each genotype correlated exclusively with the pancreatic phenotype. Quantitative data of acinar and ductular secretion were available in 93 patients. Patients with mutations belonging to classes I, II, and III had greatly reduced acinar and ductular function compared with those with class IV or $\mathrm{V}$ mutations.

Conclusion: The predicted or known functional consequences of specific mutant alleles correlate with the severity of pancreatic disease in cystic fibrosis.
\end{abstract}

ystic fibrosis (CF) affects multiple organs, including the pancreas, intestine, sweat gland, and the respiratory tract. Identification of the cystic fibrosis gene ${ }^{1-3}$ led to the elucidation of its protein product, the cystic fibrosis transmembrane conductance regulator (CFTR), a cAMP regulated chloride channel. ${ }^{4}$ Mutations in the CFTR gene result in absent or defective CFTR chloride channel function at the apical surface of epithelial cells. The most common disease causing mutation, which accounts for $66-70 \%$ of CF chromosomes worldwide, gives rise to a deletion of a single amino acid, phenylalanine, at position $508(\Delta \mathrm{F} 508)$ of the CFTR product. ${ }^{2}$ More than 1000 additional mutations (http// www.genet.sickkids.on.ca/cftr/mutations.html) or putative mutations in the CFTR gene have been identified, all of which are uncommon or rare.

Infants with $\mathrm{CF}$ have progressive pancreatic damage leading to signs and symptoms of maldigestion due to pancreatic insufficiency (PI). However, $10-15 \%$ of patients retain adequate pancreatic reserve to permit normal digestion without enzyme therapy. ${ }^{6}$ In general, those with the "pancreatic sufficient" (PS) phenotype have less severe disease manifestations. ${ }^{6}$ We have previously shown that there is a strong clinical association between phenotype and genotype in the exocrine pancreas. ${ }^{78}$ Initial investigations into the phenotype-genotype relationships in CF were made by studying the clinical phenotype and then making an assumption regarding the genotype. For example, R117H was noted to be present in PS patients and therefore an assumption was made that this was a mild mutation. ${ }^{9}$ From these studies it was determined that most individuals with PI have two severe CFTR mutations while those with the PS phenotype have one or two mild mutations. However, this correlation was limited as it was not based on the molecular mechanisms of the specific mutations. In this study, we attempt to overcome this limitation by classifying the CFTR mutations based on their functional consequences, and then evaluating the relationship with the pancreatic phenotype.
CFTR gene mutations can be classified according to their predicted effects on CFTR mediated anion secretion. ${ }^{9-11}$ In a five class system, mutations belonging to classes I, II, and III are predicted to have severe functional consequences on CFTR function via different molecular mechanisms. Mutations belonging to classes IV and V on the other hand are expected to confer some residual CFTR mediated channel function and to have milder consequences.

It is our hypothesis that the molecular consequences of CFTR gene mutations correlate with the exocrine pancreatic phenotype in patients with CF. The purpose of this analysis was to validate previous studies of the phenotype-genotype relationship by starting from the basic molecular mechanisms and then evaluating the link with the clinical phenotype. In this report, we present unique data describing the relationship between the predicted consequences of mutations of the CFTR gene and quantitative exocrine pancreatic disease in patients with CF.

\section{METHODS}

\section{Patient ascertainment}

We assessed all patients with CF attending the Toronto clinics who had undergone genotype analysis. The diagnosis of CF was based on the 1998 consensus conference criteria. ${ }^{12}$ Each patient's clinical data and genotype had been recorded in a computerised database. Each patient's pancreatic status (PS or PI) at diagnosis and subsequent changes in pancreatic status $(\mathrm{PS} \rightarrow \mathrm{PI})$ were defined. Patients who had undergone pancreatic stimulation testing were selected for further analysis of the relationship between exocrine pancreatic function and

Abbreviations: CF, cystic fibrosis; CFTR, cystic fibrosis transmembrane regulator; $\mathrm{PI}$, pancreatic insufficiency; PS, pancreatic sufficiency. 


\begin{tabular}{|c|c|c|c|c|}
\hline & $\mathrm{PI}$ & $\mathrm{PS} \rightarrow \mathrm{PI}$ & PS & p Value* \\
\hline $\operatorname{Sex}(M / F)$ & $348 / 262$ & $14 / 8$ & $60 / 50$ & 0.7 \\
\hline Age at diagnosis $(y)$ & $2.3(4.9)$ & $3.1(6.5)$ & $9.8(9.5)$ & 0.001 \\
\hline Sweat chloride (mmol/l) & $103(14)$ & $99(18)$ & $87(21)$ & 0.001 \\
\hline Age at assessment (y) & $21.0(10.4)$ & $22.6(8.4)$ & $27.3(11.7)$ & 0.03 \\
\hline $\mathrm{FEV}_{1}$ (\% predicted) & $62.0(29.4)$ & $65.7(25.9)$ & $76.2(28.7)$ & 0.001 \\
\hline Weight (\% ideal for height) & $99.8(14.6)$ & $99.9(16.3)$ & $108.8(18.3)$ & 0.001 \\
\hline Mortality (\%) & 16.6 & 9.1 & 4.6 & 0.001 \\
\hline
\end{tabular}

genotype. The study was approved by the institutional ethics review board.

\section{Assessment of exocrine pancreatic function}

Pancreatic function was assessed by one or more of the following methods.

\section{Faecal fat}

Three to five day pooled stool collections were stored at $4{ }^{\circ} \mathrm{C}$, and faecal fat content was determined by the method of van de Kamer and Weyers ${ }^{13}$ or by the method of Jeejeebhoy and Kozak. ${ }^{14}$ Faecal fat losses, expressed as a percentage of mean daily fat intake, were calculated by weighing food intake and by reference to standard food content tables. PI was defined as faecal fat losses exceeding $7 \%$ of measured fat intake in patients older than six months, or exceeding $15 \%$ in infants younger than six months of age. ${ }^{15}$

\section{Serum cationic trypsinogen}

This radioimmunoassay test reliably distinguishes patients with PS from those with PI after seven years of age, as previously described. ${ }^{16}$ PS patients with CF have shown widely fluctuating values within or above the normal range. Infants with PI have high serum trypsinogen levels which decline to low or undetectable levels by seven years of age. Patients who are PS at diagnosis but progress to PI $(\mathrm{PS} \rightarrow \mathrm{PI})$ show a progressive but delayed decline in serum trypsinogen levels. ${ }^{17}$ Patients with PS were tested every 6-12 months. If serum trypsinogen values were declining, or if clinical symptoms suggested pancreatic failure, confirmatory studies (fat balance and/or pancreatic stimulation tests) were performed.

\section{Quantitative pancreatic stimulation test}

Patients were assessed by a marker perfusion technique. ${ }^{18}$ Enzymes (colipase, total lipase, and trypsin) ${ }^{18}$ and fluid ${ }^{19}$ and electrolyte $\left(\mathrm{Cl}^{-} \mathrm{Na}^{+}, \mathrm{HCO}^{-}\right)^{20}$ output were expressed per $\mathrm{kg}$ body weight/hour after correcting for the fractional recovery of infused marker. Patients were defined as PI if colipase output was less than $120 \mathrm{u} / \mathrm{kg}$ body weight/hour or if trypsin output was below $50 \mathrm{u} / \mathrm{kg}$ body weight/hour. If several tests were performed, the results of the most recent test were used.

\section{Genotype analysis}

Genotype analysis was performed using total human genomic DNA extracted from peripheral blood cells by standard methods. Each exon of the CFTR gene was amplified by the polymerase chain reaction and screened by SSCP and MDE gel analyses. ${ }^{21}$ Direct sequencing analysis was used to characterise the DNA sequence of a suspected mutation. Based on retrospective data, this method is capable of detecting more than $95 \%$ of known CFTR gene mutations. This detection rate does not include patients with rare unknown mutations or mutations that may lie outside the boundaries of the CFTR gene (that is, in regulatory elements).

\section{Genotype classification}

Genotypes were classified according to their known or predicted functional consequences. ${ }^{9-11}$ With the exception of patients who were homozygous for $\Delta \mathrm{F} 508$, most patients were compound heterozygotes with mutations belonging to more than one class. We used the functional class of the second mutation on a $\Delta$ F508 background. This approach was used to evaluate the effect of the second mutation in patients carrying one $\Delta$ F508 mutation. For example, a patient carrying a class I mutation (for example, G542X) in combination with $\Delta \mathrm{F} 508$ (class II) was classified as class I although the genotype is in fact a compound heterozygote of class I/II. As class IV or V mutations have a dominant effect, patients with these mutations were classified according to the presumed consequences of the dominant mutation.

\section{Statistical analysis}

Patient characteristics are presented as mean (SD) and groups were compared using the Student's $t$ test; $\chi^{2}$ tests were used to compare proportions. Results of the quantitative pancreatic stimulation test produced several measurements with skewed distributions and unequal variance estimates in the subgroups to be compared. Therefore, the Kruskal-Wallis rank sum test ${ }^{22}$ was used to test subgroup differences in pancreatic secretion measures.

\section{RESULTS}

\section{Patients}

We assessed 742 patients with a confirmed clinical diagnosis of CF who had been followed between 1990 and 1997 (table 1). At diagnosis, 610 (82.2\%) were PI and 110 (14.8\%) have remained PS. Twenty two patients, who were PS at diagnosis, became PI during follow up (PS $\rightarrow$ PI). As previously observed ${ }^{67}$ patients in the PS group were diagnosed at a later age, had lower mean sweat chloride values, better pulmonary function, better nutritional status, and a lower mortality than those with the PI phenotype. There was no significant difference in sweat chloride, forced expiratory volume in one second, weight for height, or age of diagnosis between the PI and PS $\rightarrow$ PI groups.

\section{Genotype analysis}

CFTR gene mutations were identified on both alleles in 633 patients $(85.3 \%)$ and on one allele in only $95(12.8 \%)$ patients. Among the group with only one identified mutation, 40 were PS at diagnosis. No CFTR gene mutations were identified on either allele in $14(1.9 \%)$ patients, of whom seven were PS at diagnosis. Seventy six different CFTR gene mutations were identified among the 1324 chromosomes in 662 families (table 2). The most common mutations were: $\Delta \mathrm{F} 508$ (in 943 chromosomes, 71.2\%), G551D (39, 2.9\%), G542X (31, 2.3\%), $621+1 G \rightarrow T$, W1282X (16, 1.2\%), and R117H (11, 0.8\%). Less common mutations included G85E and $5 \mathrm{~T}(\mathrm{n}=5$ chromosomes), A455E and R1162X ( $\mathrm{n}=4$ chromosomes), R347, Y1092X, R334W, and V520F ( $\mathrm{n}=3$ chromosomes). In $46 \mathrm{CF}$ 
Table 2 Genotype classification according to the functional consequences of CFTR gene mutations

\begin{tabular}{|c|c|c|c|c|c|}
\hline $\begin{array}{l}\text { Pancreatic } \\
\text { status }\end{array}$ & Class I & Class II & Class III & Class IV & Class V \\
\hline PS & $\mathrm{F}^{1}, 875+1 \mathrm{G} \rightarrow \mathrm{C}(2)$ & $F, F(1)$ & $\begin{array}{l}F, G 551 D(1) \\
F, G 85 E^{2}(1)\end{array}$ & $\begin{array}{l}\mathrm{F}, \mathrm{R} 117 \mathrm{H}(11) \\
\mathrm{F}, \mathrm{R} 347 \mathrm{H}(3) \\
\mathrm{F}, \mathrm{S} 1251 \mathrm{~N}(2) \\
\mathrm{F}, \mathrm{D} 614 \mathrm{G}(1) \\
\mathrm{F}, \mathrm{R} 347 \mathrm{P}(1) \\
\text { R1 17H,R } 117 \mathrm{H}(1) \\
\mathrm{F}, \mathrm{L} 1335 \mathrm{P}(1) \\
\mathrm{F}, \mathrm{P} 67 \mathrm{~L}(1) \\
\text { F,R347P/R347H (1) } \\
\text { F,V232D(2) } \\
\text { R334W, R334W(1) }\end{array}$ & $\begin{array}{l}\mathrm{F}, 3849+10 \mathrm{kbC} \rightarrow \mathrm{T}(5) \\
\mathrm{F}, 3272-26 \mathrm{~A} \rightarrow \mathrm{G}(4) \\
\mathrm{F}, \mathrm{A} 445 \mathrm{E}(3) \\
\mathrm{F}, \mathrm{P} 574 \mathrm{H}(2) \\
\mathrm{F}, 3120 \mathrm{G}>\mathrm{A}(1) \\
\mathrm{F}, 5 \mathrm{~T}(8) \\
\mathrm{F}, 2789+5 \mathrm{G} \rightarrow \mathrm{A}(1)\end{array}$ \\
\hline $\mathrm{PS} \rightarrow \mathrm{PI}$ & $\begin{array}{l}F, 3659 \text { delC (1) } \\
F, 2184 \text { insA (1) }\end{array}$ & $F, F(15)$ & $\begin{array}{l}\text { F,G551D (1) } \\
\text { F,R560T (1) }\end{array}$ & $F, 11234 V(1)$ & \\
\hline $\mathrm{PI}$ & 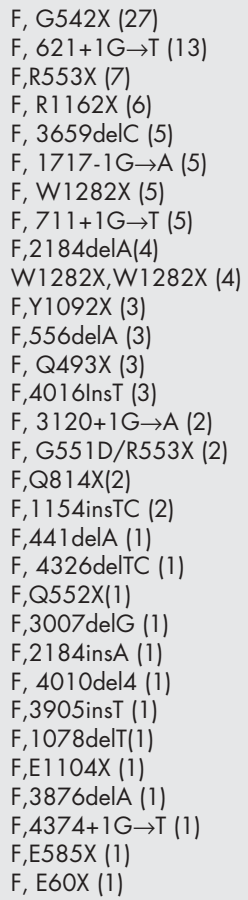 & $F, F(365)$ & $\begin{array}{l}\text { F, G551D (28) } \\
\text { F, R560T (7) } \\
\text { F, N1303K (9) } \\
\text { F,L1077P (2) } \\
\text { F, I48T (1) } \\
\text { F,A559T (1) } \\
\text { F, G85E (2) } \\
\text { G551D,G551D(1) } \\
\text { F,H199R (1) } \\
\text { F,I1072T(1) } \\
\text { F,S549 (R75Q) (1) }\end{array}$ & & \\
\hline
\end{tabular}

chromosomes a mutation was found that was absent in other tested families, and in 116 CF chromosomes no mutation was detected.

Analysis of 633 patients with two identified CFTR gene mutations confirmed our previously documented association between pancreatic phenotype and severe and mild genotypes, ${ }^{810}$ and provided further information concerning the phenotypic effects of more recently identified gene mutations. Mutations were classified as mild or severe, as previously described. ${ }^{7}$ With very few exceptions, two severe mutations conferred PI and one mild mutant allele conferred PS in a dominant manner. Among the 569 patients carrying two severe mutations, 546 (96.0\%) were PI, while 61/62 (98\%) with at least one mild mutation were PS. A subset of patients with severe mutations on both alleles were PS at diagnosis but progressed to PI. Only four individuals $(0.7 \%)$ with two severe mutations have remained PS. Pancreatic stimulation testing of one patient ( $\Delta \mathrm{F} 508 / \Delta \mathrm{F} 508)$ revealed markedly reduced pancreatic acinar function, close to the threshold for developing PI. ${ }^{18}$ The three remaining patients were not tested.

\section{Functional consequences of CFTR gene mutations and pancreatic phenotype}

We were able to classify 607 of the 633 patients according to the predicted consequences of their CFTR gene mutations (table 2). Patients who did not carry $\Delta$ F508 but who were compound heterozygotes for mutations in different classes were excluded. Ninety nine per cent (552/557) of patients with both mutations homozygous or compound heterozygous for classes I, II, or III were PI at diagnosis (528), or progressed to PI after diagnosis. ${ }^{19}$ Only five individuals with class I, II, or III mutations have remained PS since diagnosis. This includes one patient who carries the missense mutation G85E which appears to have an indeterminate effect on pancreatic phenotype as it has been observed in patients with PS and PI phenotypes. ${ }^{23}$ All but one of 49 individuals carrying at least one mutation belonging to classes IV or V have remained PS. The exception, a patient with the class IV mutation I1234V, developed PI at the age of 25 years, after symptoms of chronic pancreatitis lasting 10 years. 
Table 3 Pancreatic ductular and acinar secretion classified according to the functional consequences of CFTR gene mutations

\begin{tabular}{|c|c|c|c|c|c|c|c|}
\hline \multirow[b]{2}{*}{$\begin{array}{l}\text { Functional } \\
\text { class }\end{array}$} & \multirow[b]{2}{*}{$\mathrm{n}$} & \multicolumn{3}{|c|}{ Ductular function } & \multicolumn{3}{|c|}{ Acinar function } \\
\hline & & $\begin{array}{l}\text { Fluid } \\
(\mathrm{ml} / \mathrm{kg} / \mathrm{h})\end{array}$ & $\begin{array}{l}\text { Bicarbonate } \neq \\
(\mathrm{mmol} / \mathrm{kg} / \mathrm{h})\end{array}$ & $\begin{array}{l}\text { Chlorideł } \\
\text { (mmol/kg/h) }\end{array}$ & $\begin{array}{l}\text { Trypsin* } \\
\text { (U/kg/h) }\end{array}$ & $\begin{array}{l}\text { Colipase* } \\
\text { (U/kg/h) }\end{array}$ & $\begin{array}{l}\text { Total lipase* } \\
\text { (U/kg/h) }\end{array}$ \\
\hline Class I & 7 & $2.3(1.5)$ & $0.03(0.02)$ & $0.15(0.14)$ & $19(42)$ & $142(201)$ & 290 (388) \\
\hline Class II & 33 & $2.9(2.4)$ & $0.04(0.04)$ & $0.21(0.33)$ & 31 (63) & 111 (197) & $202(276)$ \\
\hline Class III & 6 & $1.1(0.87)$ & $0.02(0.02)$ & $0.12(0.07)$ & 109 (256) & 235 (483) & 608 (1280) \\
\hline Class IV & 14 & $4.0(2.8)$ & $0.19(0.2)$ & $0.32(0.12)$ & 1032 (768) & 9840 (10698) & 12563 (10461) \\
\hline Class V & 10 & 4.8 (3.2) & 0.12 (0.07) & $0.36(0.23)$ & 1535 (1406) & 12161 (13550) & 16449 (15482) \\
\hline Control & 21 & $10.0(3.9)$ & $0.57(0.22)$ & $0.62(0.3)$ & $2291(836)$ & $13036(5958)$ & 19767 (8623) \\
\hline \multicolumn{8}{|c|}{ 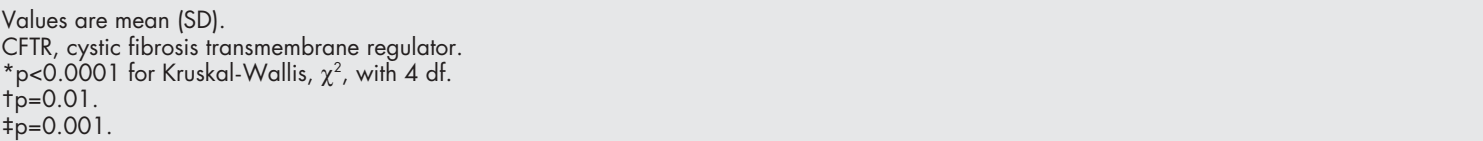 } \\
\hline
\end{tabular}
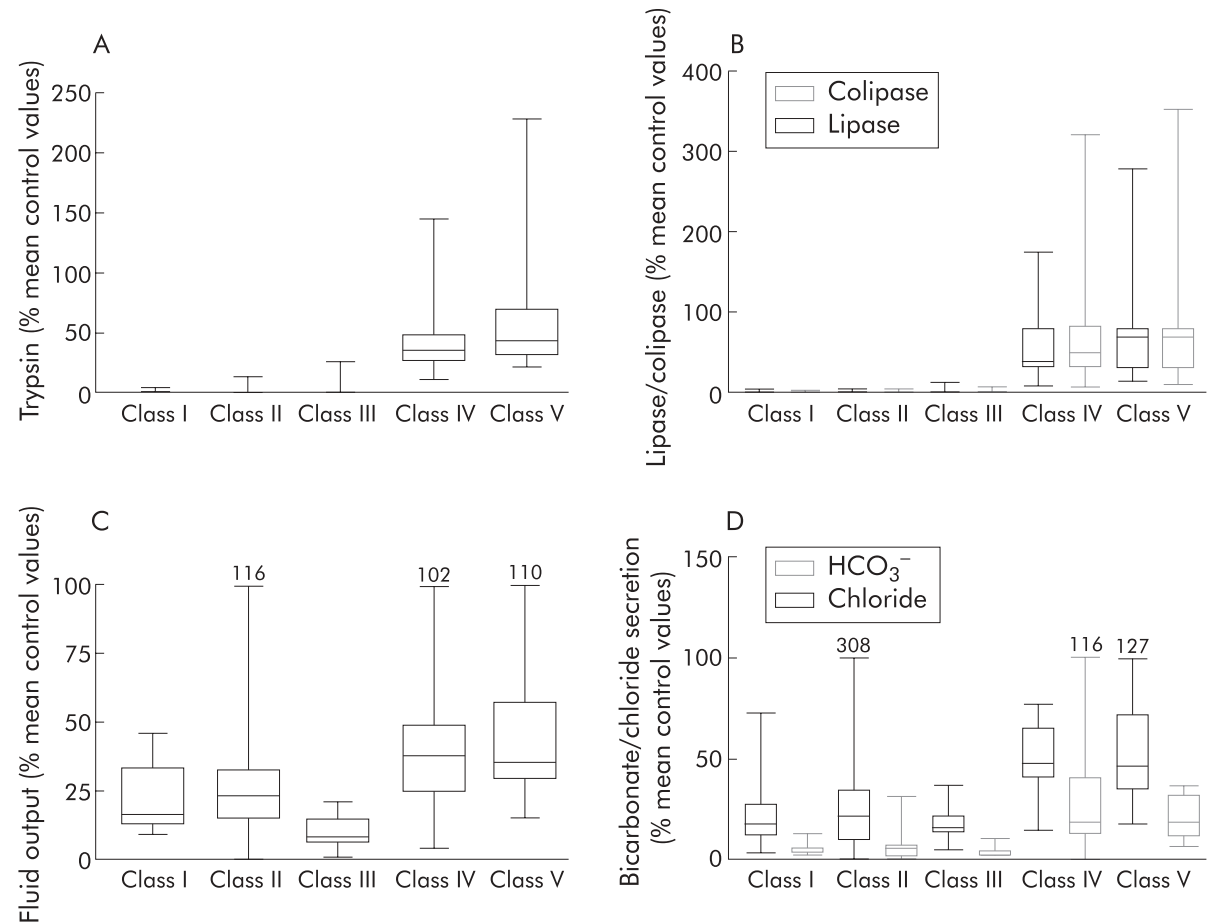

Figure 1 Quantitative pancreatic acinar and ductular secretion following intravenous cholecystokinin/secretin infusion in 70 patients, expressed as a percentage of mean control values. (A) Trypsin; (B) lipase and colipase; (C) fluid output; and (D) bicarbonate ( $\mathrm{HCO}_{3}{ }^{-}$) and chloride secretion are shown. Box margins represent the 25th and 75th percentiles, with the horizontal bar representing the median. Whiskers indicate the range of data. Comparison of classes I, II, and III with classes IV and V showed significant differences: $p=0.01$ (fluid), $p=0.001$ (bicarbonate and chloride), $\mathrm{p}<0.0001$ (trypsin, colipase and lipase) by Kruskal-Wallis, $\chi^{2}$, with $4 \mathrm{df}$.

\section{Genotypes and quantitative measures of pancreatic function}

Pancreatic function testing was performed in 95 patients (34 female), including 46 who have remained PS and 49 who were either PI at diagnosis or developed PI after diagnosis. Table 3 shows pancreatic acinar and ductular function data according to the functional classification system for CFTR gene mutations. All patients who were homozygous or compound heterozygous for mutations belonging to classes I, II, or III had severely impaired pancreatic acinar and ductular function. When expressed as a percentage of mean output reference values (fig l) there was a marked deficit of acinar capacity in patients with class I, II, or III mutations compared with those with class IV or V mutations ( $p<0.0001$, Kruskal- Wallis, $\chi^{2}, 4$ df). Among patients with class IV and V mutations, acinar function spanned a wide range of enzyme output from $18 \%$ of mean control values to values well within the reference range. Similarly, ductular function (fluid, bicarbonate, and chloride secretion) was significantly lower among patients homozygous or compound heterozygous for class I, II, or III mutations than that observed in patients with mutations belonging to class IV or V $\left(\mathrm{p}<0.01\right.$ for $\mathrm{H}_{2} \mathrm{O} ; \mathrm{p}<0.001$ for $\mathrm{HCO}^{-}$and $\left.\mathrm{Cl}^{-}\right)$. Patients in classes I, II, and III who were PS $\rightarrow$ PI were indistinguishable from those who were PI at diagnosis. Acinar output in the PS $\rightarrow$ PI group ranged from $0 \%$ to $14.3 \%$ of control values.

\section{DISCUSSION}

Attempts to elucidate the relation between genotype and the various phenotypic manifestations of CF have been hampered by the large number of CFTR gene mutations. In this study, the frequency of the most common disease causing mutation $(\Delta \mathrm{F} 508)$ was very similar to the frequency of approximately $70 \%$ of all CFTR mutant alleles reported worldwide. The four next most common mutations reported in this study (G551D, G542X, 62l $+\mathrm{lG} \rightarrow \mathrm{T}$, and W1282X) each accounted for 
between $1 \%$ to $3 \%$ of the mutations analysed. Twenty five additional mutations, including the 5 thymidine variant in intron $8{ }^{24}$ were identified in more than two families. Thus 31 CFTR gene mutations or variants which were identified in more than one family accounted for $87.8 \%$ of all mutant alleles. Forty five additional mutations, or putative mutations, which were identified in single patients, accounted for only $3.5 \%$ of mutant alleles. Despite our attempts to screen all exons of the CFTR gene, $8.8 \%$ of mutant alleles remained unidentified. These results are consistent with the recently published CFTR mutation frequencies in the French population. ${ }^{25}$

Recognising the frequency of uncommon or rare mutations in $\mathrm{CF}$, we evaluated the relation between genotype and pancreatic phenotype by classifying individual patients' CFTR gene mutations. In this analysis, no assumptions were made about the genotype based on the prior knowledge of the pancreatic phenotype, as genotypes were classified purely based on their known or predicted consequences on CFTR mediated anion secretion (fig 1). CFTR gene mutations belonging to classes I, II, or III have varying effects on gene transcription, mRNA translation, or intracellular trafficking of the nascent protein. In functional terms, however, they would all be expected to confer complete loss of cAMP regulated chloride channel function. The results of the present study conclusively indicate that homozygosity or compound heterozygosity for mutations in classes I, II, and/or III is strongly associated with severe pancreatic disease as the vast majority of these patients were PI at diagnosis or progressed to PI after the diagnosis of CF was established. By contrast, class IV and V mutations are predicted to be less severe in terms of their effects on cAMP regulated chloride channel properties. In our study, all patients carrying at least one class IV or class V mutation were PS at diagnosis, as measured by pancreatic function testing, and all but one have remained PS. The single exception appeared to develop PI due to progressive pancreatic damage from chronic pancreatitis. In support of these results, data from the European Epidemiology Registry of Cystic Fibrosis showed that patients with class IV and V mutations were less likely to be taking pancreatic enzyme supplements. However, pancreatic function status was not objectively defined..$^{26}$

A subset of patients who had undergone pancreatic stimulation testing were also evaluated according to the predicted consequences of their CFTR gene mutations. Not surprisingly, patients who were homozygous or compound heterozygous for mutations belonging to classes I, II, or III had severely compromised acinar capacity (enzyme secretion of trypsin, total lipase, and colipase). Among the PS $\rightarrow$ PI patients who were PS when tested $(n=14)$, enzyme secretion was also severely compromised (approximating $0-14.3 \%$ of mean control values). The majority of these patients, who carried severe CFTR gene mutation on both alleles, developed PI within two years of life. In contrast, patients in this study with at least one mild class IV or V mutation had significantly better pancreatic acinar reserve, which in the majority has been maintained for decades. Trypsin secretion in this group ranged from $11 \%$ of mean control values to values within our adult reference range. ${ }^{18}$

Pancreatic ductular secretion of anions (chloride and bicarbonate) and fluid was compromised in patients with CFTR gene mutations belonging to all five functional classes. This was expected because CFTR is normally expressed at high levels in the intralobular and proximal ductal epithelia, and at low levels in acinar cells. ${ }^{27}{ }^{28}$ CFTR in the ductal epithelium facilitates the secretion of an alkaline rich fluid which maintain the solubility of secreted enzymes. Complete loss of CFTR function results in reduced intraluminal $\mathrm{pH}$ and a low secretory volume..$^{29}$ Scheele and colleagues ${ }^{29}$ have suggested that reduced $\mathrm{pH}$ within the acinar lumen inhibits acinar endocytosis of secretory granule proteins and reduces the solubility of secreted luminal proteins within the acinar lumen. Complete loss of CFTR function, due to mutations on both alleles (classes I, II, or III) appears to induce rapid pancreatic atrophy through obstruction of secreted proteins within the lumina of acini and small ducts. Postmortem studies of the CF pancreas from preterm infants and neonates clearly demonstrate that this process begins in utero ${ }^{30}$ and explains the rapid progression of pancreatic failure in early infancy. Patients carrying at least one mutant allele belonging to classes IV or V also had evidence of ductular dysfunction. Chloride, bicarbonate, and fluid secretion was significantly less than that observed in adult controls even in those with normal acinar reserve. This observation supports our previous hypothesis that a major component of anion and fluid secretion is directly attributable to functional CFTR in pancreatic ductal epithelia. ${ }^{19}{ }^{20}$ Obviously, mild class IV or V CFTR gene mutations, which result in some residual ductular function, appear to protect the exocrine pancreas from complete destruction at an early age.

There are several possible explanations for the heterogeneity of acinar reserve and ductular dysfunction among patients carrying class IV and V mutations. Specific mutant alleles belonging to classes IV and V may exhibit varying degrees of chloride channel dysfunction, which in turn may influence disease progression. Secondly, patients carrying at least one class IV or V mutation may develop progressive pancreatic damage as a consequence of chronic pancreatitis. While the prevalence of acute recurrent pancreatitis or chronic pancreatitis is low in patients with CF diagnosed on clinical grounds, ${ }^{31}$ those with the PS phenotype appear to carry a high risk of this complication. ${ }^{3}$

The limitations of this classification system are acknowledged. Classification is not possible if a patient carries a "putative" mutation or if the molecular properties of a disease causing mutation are not understood. Furthermore, despite extensive efforts to identify all CFTR gene mutations, one or two mutant alleles remain unidentified in $17 \%$ of patients. A small number of mutations (for example, G85E) are considered to be indeterminate due to their inconsistent effect on pancreatic phenotype. Finally, it is more difficult to predict the functional consequences in compound heterozygotes with mutant alleles belonging to two different classes. Despite these limitations, we have overcome previous assumptions involved in analysing the relationship between genotype and phenotype in CF by starting our analysis with the predicted or known molecular consequences of the specific CFTR mutations. We have demonstrated a strong relation between the predicted functional effects of each genotype and pancreatic phenotype in this patient cohort. Nevertheless, there were a few exceptions. For example, two siblings with a class I mutation $(875+1 G>C)$ had a PS phenotype. The precise reason for this is unclear as $875+1 \mathrm{G}>\mathrm{C}$ adversely affects the highly conserved splice donor site sequence. One possibility is that alternative splicing nearby may preserve the effect of this mutant allele.

In conclusion, we have demonstrated, using analysis of the molecular consequences of CFTR mutations, that the CF genotype strongly correlates with the pancreatic phenotype. Furthermore, we provide unique data indicating that CFTR gene mutations correlate with quantitative measures of pancreatic acinar and ductular function.

\section{ACKNOWLEDGEMENTS}

Supported by grants in aid from the Canadian Cystic Fibrosis Foundation and the National Institute of Health (NIDDK-DK 49096). Dr Ahmed is the recipient of a research fellowship from the Medical Research Council (Canada)

\section{Authors' affiliations}

N Ahmed, G Forstner, Division of Gastroenterology and Nutrition, The Hospital for Sick Children, and Department of Pediatrics, University of 
Toronto, Toronto, Canada

M Corey, Program in Population Health Science, The Research Institute, The Hospital for Sick Children, and Department of Population Health Science, University of Toronto, Toronto, Canada

J Zielenski, L-C Tsui, Program in Genetics and Genomic Biology, The Research Institute, The Hospital for Sick Children, and Department of

Medical and Molecular Genetics, University of Toronto, Toronto, Canada

L Ellis, Division of Gastroenterology and Nutrition, The Hospital for Sick Children, Toronto, Canada

E Tullis, Division of Respirology, St Michael's Hospital, and Department of Medicine, University of Toronto, Toronto, Canada

P Durie, Program in Integrative Biology, The Research Institute and the Division of Gastroenterology and Nutrition, The Hospital for Sick

Children, and Department of Pediatrics, University of Toronto, Toronto,

Canada

\section{REFERENCES}

1 Riordan JR, Rommens JM, Kerem B, et al. Identification of the cystic fibrosis gene: cloning and characterization of complementary DNA. Science 1989;245: 1066-73.

2 Rommens JM, lannuzzi MC, Kerem B, et al. Identification of the cystic fibrosis gene: chromosome walking and jumping. Science 1989:245: 1059-65.

3 Kerem B, Rommens JM, Buchanan JA, et al. Identification of the cystic fibrosis gene: genetic analysis. Science 1989;245:1073-80.

4 Kartner N, Hanrahan JW, Jensen TJ, et al. Expression of the cystic fibrosis gene in non-epithelial invertebrate cells produces a regulated anion conductance. Cell 1991:64:681-91.

5 di Sant'Agnese PA. Fibrocystic disease of the pancreas with normal or partial pancreatic function. Pediatrics 1955;15:683-96.

6 Gaskin K, Gurwitz D, Durie P, et al. Improved respiratory prognosis in patients with cystic fibrosis with normal fat absorption. J Pediatr 1982; 100:857-62

7 Kerem E, Corey M, Kerem BS, et al. The relation between genotype and phenotype in cystic fibrosis - analysis of the most common mutation (delta F508). N Engl J Med 1990;323:1517-22.

8 Kristidis P, Bozon D, Corey M, et al. Genetic determination of exocrine pancreatic function in cystic fibrosis. Am J Hum Genet 1992:50: 1 178-84.

9 Welsh MJ, Smith AE. Molecular mechanisms of CFTR chloride channel dysfunction in cystic fibrosis. Cell 1993;73:1251-4.

10 Zielenski J, Tsui LC. Cystic fibrosis: genotypic and phenotypic variations. Annu Rev Genet 1995;29:777-807.

11 Wilschanski M, Zielenski J, Markiewicz D, et al. Correlation of sweat chloride concentration with classes of the cystic fibrosis transmembrane conductance regulator gene mutations. J Pediatr 1995; 127:705-10.

12 Rosenstein BJ, Cutting GR. The diagnosis of cystic fibrosis: a consensus statement. Cystic Fibrosis Foundation Consensus Panel. J Pediatr 1998:132:589-95.

13 Van de Kamer HH, Weyers HA. Rapid method for determination of fat in feces. J Biol Chem 1949;177:347-51

14 Jeejeebhoy KN AS, Kozak G. Determination of fecal fats containing both medium and long-chain triglycerides and fatty acids. Clin Biochem 1970;3:157-63.
15 Fomon SJ, Ziegler EE, Thomas LN, et al. Excretion of fat by normal full-term infants fed various milks and formulas. Am J Clin Nutr 1970;23:1299-313.

16 Durie PR, Forstner GG, Gaskin K, et al. Age-related alterations of immunoreactive pancreatic cationic trypsinogen in sera from cystic fibrosis patients with and without pancreatic insufficiency. Pediatr Res 1986;20:209-13

17 Couper RT, Corey M, Durie PR, et al. Longitudinal evaluation of serum trypsinogen measurement in pancreatic-insufficient and pancreatic-sufficient patients with cystic fibrosis. J Pediatr 1995:1273.

18 Gaskin KJ, Durie PR, Lee L, et al. Colipase and lipase secretion in childhood-onset pancreatic insufficiency. Delineation of patients with steatorrhea secondary to relative colipase deficiency. Gastroenterology 1984;86:1-7.

19 Kopelman H, Durie P, Gaskin K, et al. Pancreatic fluid secretion and protein hyperconcentration in cystic fibrosis. N Engl J Med $1985: 312: 329-34$

20 Kopelman H, Corey M, Gaskin K, et al. Impaired chloride secretion, as well as bicarbonate secretion, underlies the fluid secretory defect in the cystic fibrosis pancreas. Gastroenterology 1988;95:349-55.

21 Zielenski J, Aznarez I, Onay T, et al. CFTR mutation detection by multiplex heteroduplex (mHET) analysis on MDE gels. In: Skach WR, ed. Cystic Fibrosis Methods and Protocols-Methods in Molecular Medicine. Totawa NJ: Humana Press, 2002:3-19.

22 Lehmann E. Nonparametrics: Statistical Methods Based on Ranks. San Francisco: Holden-Day; 1975

23 Vazquez C, Antinolo G, Casals T, et al. Thirteen cystic fibrosis patients, 12 compound heterozygous and one homozygous for the missense mutation G85E: a pancreatic sufficiency/insufficiency mutation with variable clinical presentation. J Med Genet 1996:33:820-2.

24 Chu CS, Trapnell BC, Curristin S, et al. Genetic basis of variable exon 9 skipping in cystic fibrosis transmembrane conductance regulator mRNA. Nat Genet 1993;3:151-6.

25 Claustres M, Guittard C, Bozon D, et al. Spectrum of CFTR mutations in cystic fibrosis and in congenital absence of the vas deferens in France. Hum Mutat 2000;16:143-56.

26 Koch C, Cuppens H, Rainisio M, et al. European Epidemiologic Registry of Cystic Fibrosis (ERCF): comparison of major disease manifestations between patients with different classes of mutations. Pediatr Pulmonol 2001;31:1-12.

27 Marino CR, Matovcik LM, Gorelick FS, et al. Localization of the cystic fibrosis transmembrane conductance regulator in pancreas. J Clin Invest 1991:88:712-16.

28 Zeng W, Lee MG, Yan M, et al. Immuno and functional characterization of CFTR in submandibular and pancreatic acinar and duct cells. Am J Physiol 1997;273:C442-55

29 Scheele GA, Fukuoka Sl, Kern HF, et al. Pancreatic dysfunction in cystic fibrosis occurs as a result of impairments in luminal $\mathrm{pH}$, apical trafficking of zymogen granule membranes, and solubilization of secretory enzymes. Pancreas 1996:12:1-9.

30 Imrie JR, Fagan DG, Sturgess JM. Quantitative evaluation of the development of the exocrine pancreas in cystic fibrosis and control infants. Am J Pathol 1979;95:697-707.

31 Durno C, Corey M, Zielenski J, et al. Genotype and phenotype correlations in patients with cystic fibrosis and pancreatitis. Gastroenterology 2002;123:1857-64. 